\title{
Cosmic Variance in the Transparency of the Intergalactic Medium after Reionization
}

\section{Citation}

Wyithe, J. Stuart B., and Abraham Loeb. 2006. "Cosmic Variance in the Transparency of the Intergalactic Medium after Reionization." The Astrophysical Journal 646 (2): 696-702. https:// doi.org/10.1086/502620.

\section{Permanent link}

http://nrs.harvard.edu/urn-3:HUL.InstRepos:41393448

\section{Terms of Use}

This article was downloaded from Harvard University's DASH repository, and is made available under the terms and conditions applicable to Other Posted Material, as set forth at http:// nrs.harvard.edu/urn-3:HUL.InstRepos:dash.current.terms-of-use\#LAA

\section{Share Your Story}

The Harvard community has made this article openly available.

Please share how this access benefits you. Submit a story.

Accessibility 
Draft version November 13, 2017

Preprint typeset using $\mathrm{LAT}_{\mathrm{E}} \mathrm{X}$ style emulateapj v. 11/12/01

\title{
COSMIC VARIANCE IN THE TRANSPARENCY OF THE INTERGALACTIC MEDIUM AFTER REIONIZATION
}

\author{
J. Stuart B. Wyithe ${ }^{1}$ and Abraham Loeb ${ }^{2}$ \\ swyithe@physics.unimelb.edu.au; aloeb@cfa.harvard.edu \\ Draft version November 13, 2017
}

\begin{abstract}
Following the completion of cosmic reionization, the mean-free-path of ionizing photons was set by a population of Ly-limit absorbers. As the mean-free-path steadily grew, the intensity of the ionizing background also grew, thus lowering the residual neutral fraction of hydrogen in ionization equilibrium throughout the diffuse intergalactic medium (IGM). Ly $\alpha$ photons provide a sensitive probe for tracing the distribution of this residual hydrogen at the end of reionization. Here we calculate the cosmic variance among different lines-of-sight in the distribution of the mean Ly $\alpha$ optical depths. We find fractional variations in the effective post-reionization optical depth that are of order unity on a scale of $\sim 100$ co-moving Mpc, in agreement with observations towards high-redshift quasars. Significant contributions to these variations are provided by the cosmic variance in the density contrast on the scale of the mean-free-path for ionizing photons, and by fluctuations in the ionizing background induced by delayed or enhanced structure formation. Cosmic variance results in a highly asymmetric distribution of transmission through the IGM, with fractional fluctuations in Ly $\alpha$ transmission that are larger than in $\operatorname{Ly} \beta$ transmission.
\end{abstract}

Subject headings: cosmology: theory - galaxies: formation

\section{INTRODUCTION}

It is thought that the appearance of the first galaxies and quasars reionized the cosmic hydrogen in the intergalactic medium (IGM) that was left neutral following the recombination era at $z \sim 10^{3}$. The process started with the emergence of ionized regions around galaxies and then groups of galaxies. These H II regions eventually overlapped, leaving the IGM highly ionized in low-density regions surrounding pockets of residual neutral hydrogen in high density regions. The process of reionization was inhomogeneous (Furlanetto et al. 2004), and the overlap should have occurred at different times in different regions (Wyithe \& Loeb 2004b). However the identification of a Gunn-Peterson trough in the spectra of the most distant quasars at redshifts of $z \sim 6.3-6.4$ (Fan et al. 2004) hints that the reionization of cosmic hydrogen was completed near $z \sim 6$, about a billion years after the big bang (White et al. 2003; Wyithe \& Loeb 2004a; Mesinger \& Haiman 2004).

Prior to the overlap epoch, the mean-free-path of ionizing photons was limited by the size of individual $\mathrm{H}$ II regions. Following overlap, regions of IGM were exposed to ionizing radiation from additional sources. This increase in the ionizing intensity ionized hydrogen in denser regions, and resulted in an increased mean-free-path which in turn led to an even higher ionizing intensity. As the ionizing intensity in the post-overlap IGM rose, the optical depth to Ly $\alpha$ absorption decreased (e.g. Fan et al. 2002). Observations of the absorption spectra of high redshift quasars (Fan et al. 2004) and potentially gamma-ray burst afterglows (Barkana \& Loeb 2004; Chen et al. 2005) provide clues to the evolution of the ionizing radiation field and of the evolution of the ionizing photon mean-free-path.

The post-overlap evolution in the IGM is expected to be inhomogeneous. Indeed values of optical depth to Ly $\alpha$ absorption measured along different lines of sight at $5 \lesssim z \lesssim 6$ show fluctuations that are of order unity (Becker et al. 2001; White et al. 2003; Djorgovski 2004). The Ly $\alpha$ optical depth therefore varies from place to place at any slice of cosmic time. In addition to small scale variations in Ly $\alpha$ optical depth generated by structures like filaments and mini-halos (the so-called Ly $\alpha$ forest), there would be large scale inhomogeneities in the evolution of the ionization state of the IGM. Fluctuations in the transmission averaged on a large scale are sensitive to both small scale structure along the line of sight (Lidz et al. 2005 ), and to cosmic variance on larger scales. Here we concentrate on the contribution from cosmic variance in the smoothed density field. However the presence of highcolumn density absorbers and small scale structure in the density field will add additional fluctuations to those described in this paper. Variations in the average transmission would be present on the scale of the mean-free-path for ionizing photons ( 10-100 co-moving Mpc). Indeed cosmic variance on scales of $10 \mathrm{~s}$ of Mpc can be significant and has not described by contemporary numerical simulations of the reionization process (Barkana \& Loeb 2004). To model cosmic variance properly with numerical simulations requires resolving low-mass galaxies as well as solving the radiative transfer equations inside a very large box (hundreds of co-moving Mpc) with a high dynamic range of scales. Progress is now being made toward this goal (Kohler, Gnedin, Miralda-Escudé \& Shaver 2005; Kohler, Gnedin \& Hamilton 2005; Iliev et al. 2005).

In this paper we examine the variations in the optical depth averaged over a redshift bin of sufficient size to smooth out the small-scale inhomogeneities of the Ly $\alpha$ forest. Our model for the variation in optical depth includes

${ }^{1}$ University of Melbourne, Parkville, Victoria, Australia

${ }^{2}$ Harvard-Smithsonian Center for Astrophysics, 60 Garden St., Cambridge, MA 02138 
several contributions: (i) variations in the density-contrast on the scale of the mean-free-path for ionizing photons, (ii) variations in the ionizing background on the same scale due to enhanced or delayed structure-formation, (iii) variations in the ionizing background due to finite light travel time across the clustering length of ionizing sources, (iv) variations in the ionizing background due to Poisson fluctuations in the number of ionizing sources, and $(v)$ evolution of the density of Ly-limit absorbers. Throughout the paper we adopt the set of cosmological parameters determined by the Wilkinson Microwave Anisotropy Probe (WMAP, Spergel et al. 2003), namely mass density parameters of $\Omega_{m}=0.27$ in matter, $\Omega_{b}=0.044$ in baryons, $\Omega_{\Lambda}=0.73$ in a cosmological constant, and a Hubble constant of $H_{0}=71 \mathrm{~km} \mathrm{~s}^{-1} \mathrm{Mpc}^{-1}$.

\section{FLUCTUATIONS IN IONIZING INTENSITY AND LY $\alpha$ OPTICAL DEPTH}

The background intensity of ionizing radiation $J_{\nu}$ is proportional to the co-moving luminosity density due to ionizing sources $\left(n_{\mathrm{src}}\right)$, the density contrast $(\Delta)$, the bias of sources in an overdense region $\left(B_{\mathrm{src}}\right)$, the co-moving meanfree-path for ionizing photons $\left(R_{\mathrm{mfp}}\right)$, and a factor $\left(F_{\mathrm{Q}}\right)$ describing the fractional contribution of quasars to the ionizing back-ground relative to stars

$$
J_{\nu} \propto n_{\mathrm{src}}(1+z)^{3} \Delta B_{\mathrm{src}} \frac{R_{\mathrm{mfp}}}{1+z}\left(1+F_{\mathrm{Q}}\right) .
$$

Note that we assume a universal spectrum for the contribution to $J_{\nu}$ arising from a fixed redshift because we are averaging over a large number of sources. However the contribution to the ionizing background at a fixed point in the IGM from redshifted photons will result in a value for $J_{\nu}$ that depends on the shape of this universal spectrum, as well as the mean-free-path. On the other hand this dependence on contribution from redshifted photons is the same in all regions of size $R_{\mathrm{mfp}}$. Therefore since in this paper our interest lies in relative fluctuations of the ionizing back-ground among random regions of fixed size centered at the same redshift, we assume the exact shape of the spectrum to be unimportant for our calculation.

The mean-free-path $\left(R_{\mathrm{mfp}}\right)$ is in turn related to the effective co-moving density of Ly-limit absorbers $\left(\Delta \times n_{\text {abs }}\right)$ as well as their bias $\left(B_{\text {abs }}\right)$

$$
\frac{R_{\mathrm{mfp}}}{(1+z)} \propto\left[n_{\mathrm{abs}}(1+z)^{3} \Delta \times B_{\mathrm{abs}}\right]^{-1} .
$$

We can also relate the average co-moving density of sources to the collapsed fraction of baryons $\left(F_{\mathrm{col}}\right)$ in dark-matter halos above some minimum mass (that reflects the threshold for cooling and fragmentation of the gas into stars or accreting black holes). We assume that ionizing photons were generated by starbursts, and therefore expect that the luminosity density of ionizing photons was proportional to the star-formation rate. We further assume that the starbursts formed primarily out of newly collapsing IGM. As a result the star-formation rate is assumed to be proportional to the derivative of the collapsed fraction $n_{\text {src }} \propto\left(d F_{\text {col }} / d t\right)$, so that

$$
J_{\nu} \propto \frac{\left(d F_{\mathrm{col}} / d t\right)}{n_{\mathrm{abs}}} \frac{B_{\mathrm{src}}}{B_{\mathrm{abs}}}\left(1+F_{\mathrm{Q}}\right) .
$$

If both sources and absorbers reside in halos of the same mass, then $B_{\mathrm{abs}}=B_{\mathrm{src}}$. This may be the case once the gas in mini-halos below the cooling and fragmentation threshold, has been photo-evaporated. However at high redshifts just after the overlap of $\mathrm{H}$ II regions, the mini-halos might provide a significant contribution to the population of Lylimit absorbers (Barkana \& Loeb 2002; Iliev, Shapiro, \& Raga 2005). In this case $B_{\mathrm{src}}>B_{\text {abs. }}$. These bias factors are expected to evolve with time; $B_{\mathrm{src}}$ may have a slow redshift dependence through a changing minimum mass for galaxy formation while $B_{\text {abs }}$ may have a value that changes with redshift if the typical absorber mass changes with time (for example if mini-halos are being evaporated) and so $B_{\text {abs }}$ could be a function of the evolving $J_{\nu}$.

Suppose that $n_{\text {abs }}$ is some general function of $F_{\text {col }}$ and $J_{\nu}$. Ly-limit systems are expected to shrink in physical cross-section as their lower-density envelopes are ionized by a growing ionizing background. The effective density of absorbers should therefore be a function of $J_{\nu}$ in addition to $F_{\text {col }}$. Moreover since $F_{\text {col }}$ and $\left(d F_{\text {col }} / d t\right)$ are both monotonic there is a unique one-to-one correspondence between the values of $F_{\text {col }}$ and $\left(d F_{\text {col }} / d t\right)$ as a function of time. Equation (3) is therefore an implicit equation for $J_{\nu}$ as a function of $F_{\text {col }}$. The above considerations lead to two interesting conclusions. First, we find that $J_{\nu}$ should be independent of density contrast at a fixed value of collapsed fraction; and second, the average ionizing background $J_{\nu}$ within a region of radius equal to $R_{\mathrm{mfp}}$ should be a function only of the value for $F_{\text {col }}$. With these points in mind we can estimate the scatter in the redshift at which a threshold value of $J_{\nu}$ is reached, and from it find the corresponding scatter in the value of $J_{\nu}$ at fixed redshift.

\subsection{Fluctuations due to cosmic variance}

Large-scale inhomogeneity in the cosmic density field leads to structure-formation that is enhanced in over-dense regions and delayed in under-dense regions. The resulting cosmic variance in the redshift at which a critical value of $F_{\text {col }}$ is reached within regions of size $R$ may be calculated as

$$
\left\langle\delta z_{\mathrm{cv}}^{2}\right\rangle^{\frac{1}{2}}=\frac{\sigma_{R}}{\delta_{c}}(1+z),
$$

where $\sigma_{R}$ is the r.m.s. amplitude of the linear density field smoothed over spheres of radius $R$, and $\delta_{c}$ is a critical overdensity for collapse $(\propto(1+z)$ at high redshift; Barkana \& Loeb 2004; Bond et al. 1991). Since the ionizing background within a given region depends on the value of the collapsed fraction, we can relate $\left\langle\delta z_{\mathrm{cv}}^{2}\right\rangle^{\frac{1}{2}}$ to the cosmic variance in redshift where a critical value of $J_{\nu}$ is obtained. The value of $J_{\nu}$ is independent of $\Delta$. We therefore use the mean-free-path averaged over all overdensities as the appropriate length scale over which the cosmic variance is calculated, i.e. $R=R_{\mathrm{mfp}} / 2$.

The left panel of Figure 1 shows the scatter in the redshift at which a critical value of $J_{\nu}$ is obtained due to cosmic variance, $\left\langle\delta z_{\mathrm{cv}}^{2}\right\rangle$ (solid lines). We would like to convert this scatter in redshift to a scatter in the optical-depth to Ly $\alpha$ absorption at a given redshift. At a fixed redshift $z$, the local value of Ly $\alpha$ optical depth $\left(\tau_{1}\right)$ scales as

$$
\tau_{1} \propto \rho_{\mathrm{HI}}^{2} J_{\nu}^{-1},
$$

where $\rho_{\mathrm{HI}}$ is the density of neutral hydrogen (Barkana \& Loeb 2004). The effective optical depth $(\tau)$ is measured 

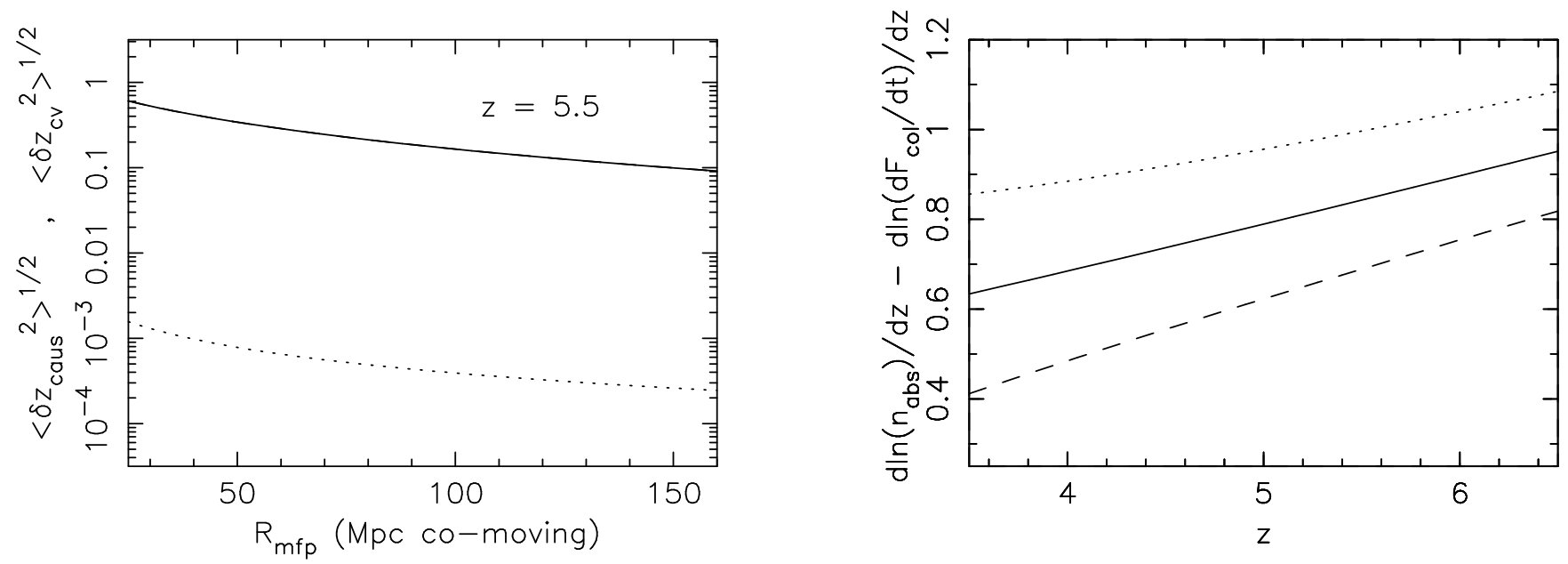

FIG. 1.- Left panel: Contribution to scatter in redshift from cosmic variance $\left\langle\delta z_{\mathrm{cv}}^{2}\right\rangle^{\frac{1}{2}}$ (solid line) and finite light travel time $\left\langle\delta z_{\text {caus }}^{2}\right\rangle^{\frac{1}{2}}$ (dotted line) as a function of the mean-free-path for ionizing photons in co-moving Mpc at a redshift of 5.5. Right panel: The value of $d \ln n_{\mathrm{abs}} / d z-d \ln \left(d F_{\mathrm{col}} / d t\right) / d z$ as a function of redshift. The dashed, solid and dotted lines correspond to $\gamma=2,3$, and 4 respectively. In calculating $F_{\text {col }}$ we have assumed a minimum virial temperature for galaxies of $2 \times 10^{5} \mathrm{~K}$.

over a path-length through the IGM, and may be computed from $\tau=-\log F$ where $F=\left\langle e^{-\tau_{1}}\right\rangle$ is the transmission averaged over the path-length. While the ionizing background will be sensitive to cosmic variance in density contrast calculated in 3-dimensional volumes $(\Delta)$ on the scale $R_{\mathrm{mfp}}$, the absorption spectra of quasars probe only lines-of-sight through these volumes. The effective optical depth averaged over a length scale $R_{\mathrm{mfp}}$ therefore depends on the square of the density averaged along the line-ofsight (Lidz, Oh \& Furlanetto 2005). Here we neglect density fluctuations on small scales and approximate the average of the density contrast squared using the smoothed density contrast $\left(\Delta_{z}\right)$ calculated in a thin cylinder at redshift $z$. This procedure underestimates the fluctuations in transmission (Lidz et al. 2005). Note that although $\tau$ varies with $z$ due to the growth factor of density perturbations, we focus here on the scatter in $\tau$ at a fixed $z$. The fractional change in the value of the optical depth relative to the average, given a density contrast $\left(\Delta_{z}\right)$, and a delay $(\delta z)$ in the redshift where the critical collapse fraction is reached is

$$
\begin{aligned}
\delta_{\tau} \equiv \frac{\tau-\tau_{\mathrm{av}}}{\tau_{\mathrm{av}}} & =\Delta_{z}^{2} \frac{J_{\nu}\left(z_{\mathrm{av}}\right)}{J_{\nu}\left(z_{\mathrm{av}}\right)+\frac{d J_{\nu}}{d z} \delta z}-1 \\
& =\Delta_{z}^{2}\left(1+\frac{d \ln J_{\nu}}{d z} \delta z\right)^{-1}-1
\end{aligned}
$$

where $\tau_{\mathrm{av}}$ is the average value of optical depth at $z_{\mathrm{av}}$. But $J_{\nu} \propto\left(d F_{\text {col }} / d t\right) n_{\text {abs }}^{-1}\left(B_{\text {src }} / B_{\text {abs }}\right)\left(1+F_{\mathrm{Q}}\right)$ [equation 3]. Within this expression we can only compute the evolution of collapsed fraction, and therefore assume an evolution specified by a parameter $\gamma$ and of the form $n_{\text {abs }}\left(B_{\text {abs }} / B_{\text {src }}\right)\left(1+F_{\mathrm{Q}}\right) \propto(1+z)^{\gamma}$ for the remaining quantities. Since we are considering a fractional change in redshift that is much smaller than unity a power-law approximation is an appropriate choice. With this addition we get

$$
\delta_{\tau}=\Delta_{z}^{2}\left(1+\left(\frac{d \ln \left(d F_{\mathrm{col}} / d t\right)}{d z}-\frac{\gamma}{(1+z)}\right) \delta z\right)^{-1}-1
$$

We note that equation (7) is a first order expression for $\delta_{\tau}$. This choice may be justified by the fact that $\left[\frac{d \ln \left(d F_{\text {col }} / d t\right)}{d z}-\frac{\gamma}{(1+z)}\right]$ has an evolution which is nearly linear with redshift (see figure 1).

At this point we specify the value of $\gamma$. One can observe the average number of Ly-limit systems per unit co-moving length along the line-of-sight $\left(R_{\mathrm{LL}}\right)$. Given the observed distribution of column densities for Ly-limit systems, this distance can be related to the mean free path for Ly-limit photons $R_{\mathrm{mfp}}=R_{\mathrm{LL}} / \sqrt{\pi}$ (MiraldaEscude 2003). Storrie-Lombardi et al. (1994) measured the abundance of Ly-limit systems per unit redshift at $z=4, d N_{\mathrm{LL}} / d z \sim 3.3$, leading to a co-moving spacing of $R_{\mathrm{LL}}=[(1+z) c(d t / d z) / 3.3]$ for Ly-limit systems along the line-of-sight. The resulting mean-free-path for Ly-limit photons is $R_{\mathrm{mfp}} \sim 120$ co-moving Mpc at $z=4$. This value provides an upper limit on the mean-free-path for Ly-limit photons at higher redshifts when the cosmic gas is denser and less ionized. The evolution of the number of Ly-limit systems per redshift is $d N_{\mathrm{LL}} / d z \propto(1+z)^{\beta}$ with $\beta \sim 1.5$ (Storrie-Lombardi et al. 1994). The evolution in the number of lines per unit co-moving length $x$ is therefore

$$
\frac{d N_{\mathrm{LL}}}{d x} \propto(1+z)^{-1} \frac{d N_{\mathrm{LL}}}{d z}\left(\frac{c d t}{d z}\right)^{-1} \propto(1+z)^{\beta+\frac{3}{2}},
$$

where $c$ is the speed of light and we have neglected the influence of the cosmological constant at high redshift. The density of absorbers is proportional to $d N_{\mathrm{LL}} / d x$, hence we find $n_{\mathrm{abs}} \propto(1+z)^{\beta+\frac{3}{2}}$, and thus $\gamma=\beta+\frac{3}{2}$. The value of $\beta$ is measured at $z=4$. However mini-halos may be important at high redshift so that an extrapolation of absorption properties from low to high redshift is not justified. We therefore consider a range of values for $\beta$ which allow for a range of evolutionary properties of the absorbers as well as their bias, and of the contribution of quasars to the ionizing background. In the right panel of Figure 1 we plot the values of the difference between $d \ln n_{\text {abs }} / d z$ and $d \ln \left(d F_{\mathrm{col}} / d t\right) / d z$ as a function of redshift for $\beta=0.5,1.5$ and $2.5(\gamma=2,3$ and 4$)$.

In a region of density contrast $\Delta$, we can compute the typical delay due to cosmic variance $\left\langle\delta z_{\text {cv }}^{2}\right\rangle^{\frac{1}{2}}$ (see equation 4). However to estimate the typical fluctuations in the 
effective optical depth we must also compute the variance in overdensity among lines of sight through the density field of length $R_{\mathrm{mfp}}$. We calculate the power-spectrum of fluctuations in cylinders of length $L$ and radius $R$ to second order in the wave number $k$ (Kaiser \& Peacock 1991; Baugh 1996)

$$
P_{1 \mathrm{~d}}(k)=\frac{1}{(2 \pi)^{2}} \frac{2 \pi}{L} \int_{k}^{\infty} d y y P_{\mathrm{b}}(y) e^{-\left(y^{2}-k^{2}\right) R^{2} / 4},
$$

where $P_{\mathrm{b}}(k)$ is the linear baryonic power-spectrum, which may be approximated as $P_{\mathrm{b}}(k)=P(k)\left(1+k^{2} R_{\mathrm{f}}^{2}\right)^{-2}$ in terms of the cold dark matter power-spectrum $P(k)$ and the filtering scale $R_{\mathrm{f}}$ for the associated reionization history (Gnedin \& Hui 1998). The variance in $\Delta_{z}$ on a scale $R$ with wavenumber $k_{\mathrm{R}}$ follows from

$$
\sigma_{R, z}=\frac{1}{2 \pi} \int_{0}^{k_{\mathrm{R}}} d k P_{1 \mathrm{~d}}(k) .
$$

The radius of the cylinder $R$ is set by the size of the quasar emission region, which is much smaller than the filtering scale. Our results are therefore insensitive to the precise choice of $R$. To estimate typical fluctuations in the optical depth from cosmic variance we assume $\Delta_{z}$ and $\Delta$ to be approximately uncorrelated and combine the 1-sigma fluctuation in delay, $\left(\left\langle\delta z_{\mathrm{cv}}^{2}\right\rangle^{\frac{1}{2}}\right)$, with the 1-sigma fluctuation in density contrast, $\left(\Delta_{z}=1 \pm \sigma_{\mathrm{R}, \mathrm{z}} D\right.$, where $D$ is the growth factor). The typical positive $(+)$ and negative (-) fluctuations in optical depth due to cosmic variance may therefore be estimated as

$$
\begin{gathered}
\left\langle\delta_{\tau, \mathrm{cv}}^{2}\right\rangle^{\frac{1}{2}}=\left(1 \pm \sigma_{\mathrm{R}, \mathrm{Z}} D\right)^{2} \\
\times\left(1 \pm\left(\frac{d \ln \left(d F_{\mathrm{col}} / d t\right)}{d z}-\frac{\gamma}{(1+z)}\right)\left\langle\delta z_{\mathrm{cv}}^{2}\right\rangle^{\frac{1}{2}}\right)^{-1}-1
\end{gathered}
$$

Figure 2 shows the typical positive $(+)$ fluctuation $\left\langle\delta_{\tau, \mathrm{cv}}^{2}\right\rangle^{\frac{1}{2}}$, which is monotonic in $R_{\mathrm{mfp}}$.

\subsection{Fluctuations due to the finite light travel time}

Following the appearance of sources with luminosity density $\Delta \times n_{\mathrm{src}}$, the ionizing intensity will grow to its full value $J_{\nu}$ over a timescale $R_{\mathrm{mfp}} /[c(1+z)]$. The time when a critical $J_{\nu}$ is achieved is affected by this light propagation delay (with ionizing sources emitting their radiation at a retarded time). However, there may also be a scatter in the time where the critical $J_{\nu}$ is achieved from place to place within the region of co-moving size $R_{\mathrm{mfp}}$. This scatter will depend on the distribution of sources. For example, if the sources were clustered on a scale similar to the mean-free-path, then within a region there will be a scatter of $R_{\operatorname{mfp}}[(1+z) c d t / d z]^{-1}$ in redshift where the critical $J_{\nu}$ is achieved. However if the clustering is on a much smaller scale then the scatter will be smaller.

We can estimate this scatter by considering the clustering length of sources, $R_{0}$ (which is $\sim 4$ co-moving Mpc for dark-matter halos with a virial temperature of $T_{\mathrm{vir}} \sim 2 \times 10^{5} \mathrm{~K}$ at $\left.z \sim 5.5\right)$. The photons must travel a distance $R_{\mathrm{mfp}}(1+z)^{-1}$ before the critical $J_{\nu}$ is reached. The scatter in arrival times for photons coming from sources clustered on a scale $R_{0}$ at a distance $R_{\mathrm{mfp}}$ may be estimated as the crossing time of the smaller clustering length $\left(R_{0} / c\right)$ divided by the square-root of the number $\left(N_{\text {cluster }}\right)$ of clustered regions at a distance $R_{\mathrm{mfp}}$ (where the last factor corresponds to Poisson fluctuations in the distances of the clustered regions). Hence the variance in redshift where the critical $J_{\nu}$ is reached due to the light propagation delay will therefore be

$$
\left\langle\delta z_{\text {caus }}^{2}\right\rangle^{\frac{1}{2}}=\frac{R_{0}}{\sqrt{N_{\text {cluster }}}(1+z)}\left(\frac{c d t}{d z}\right)^{-1},
$$

where $N_{\text {cluster }}$ is estimated as

$$
N_{\text {cluster }} \sim \frac{4 \pi\left(R_{\mathrm{mfp}} / 2\right)^{2} R_{0}}{4 \pi / 3 R_{0}^{3}}=\frac{3}{4}\left(\frac{R_{\mathrm{mfp}}}{R_{0}}\right)^{2} .
$$

The corresponding redshift variance due to the light propagation delay $\left\langle\delta z_{\text {caus }}^{2}\right\rangle^{\frac{1}{2}}$ is shown on the left panel of Figure 1. At a fixed density contrast, the finite light travel time will introduce a scatter in the value of $\delta_{\tau}$ of

$$
\begin{gathered}
\left\langle\delta_{\tau, \text { caus }}^{2}\right\rangle^{\frac{1}{2}}= \\
\left(1+\left(\frac{d \ln \left(d F_{\text {col }} / d t\right)}{d z}-\frac{\gamma}{(1+z)}\right)\left\langle\delta z_{\text {caus }}^{2}\right\rangle^{\frac{1}{2}}\right)^{-1}-1 .
\end{gathered}
$$

We find that the fluctuations in optical depth due to this effect are much smaller than those due to cosmic variance (see Figure 2).

\subsection{Fluctuations due to the finite number of ionizing sources}

The Press-Schechter formalism predicts a probability distribution (and hence a scatter) for the number of halos within a region of a given matter overdensity. In this paper we use the derivative of the collapsed fraction of baryons (up to a proportionality constant) as a proxy for the star formation rate. This quantity depends on the overdensity $\Delta$. As a result there is a scatter in the redshift where a given collapsed fraction is reached among representative regions of the universe, or equivalently there is scatter among the collapsed fractions within different regions at fixed time. In this paper we are concerned with regions of overdensity $\Delta$ and finite size $R_{\mathrm{mfp}}$. Therefore, in addition to the fluctuations in the density and ionizing radiation introduced by this cosmic variance (and by the light propagation delay), we expect a spatial variation of the ionizing intensity due to fluctuations in the number of sources within the finite region. We assume that these fluctuations may be represented by Poisson noise in the number of ionizing sources within a region of size $R_{\mathrm{mfp}}$. We estimate the number of galaxies using the Press-Schechter (1974) mass function $d n_{\mathrm{PS}} / d M$ (with the modification of Sheth \& Tormen 1999) and evaluate the number per logarithm of mass at the minimum mass for galaxy formation $\left(M_{\min }\right)$. The number of ionizing sources at a particular epoch is given by

$$
N_{\mathrm{src}}=\left.\frac{t_{\mathrm{lt}}}{t_{\mathrm{H}}} \frac{d n_{\mathrm{PS}}}{d \ln M}\right|_{M_{\min }} \frac{4 \pi}{3}\left(\frac{R_{\mathrm{mfp}}}{2}\right)^{3},
$$

where $t_{1 \mathrm{t}}$ is the source lifetime and $t_{\mathrm{H}}$ is the Hubble time at redshift $z$. In estimating the fluctuations due to variations in the source numbers we consider two limiting cases. First, we adopt $t_{\mathrm{lt}} / t_{\mathrm{H}} \sim 0.01$ corresponding to starburst lifetimes of $\sim 10^{7}$ years at $z \sim 5.5$ (the lifetimes of massive stars) and a minimum mass corresponding to the Jeans mass in a photo-ionized IGM (halo virial temperature of $\left.\sim 2 \times 10^{5} \mathrm{~K}\right)$. This case corresponds to the smallest source numbers and hence the largest level of Poisson fluctuations 


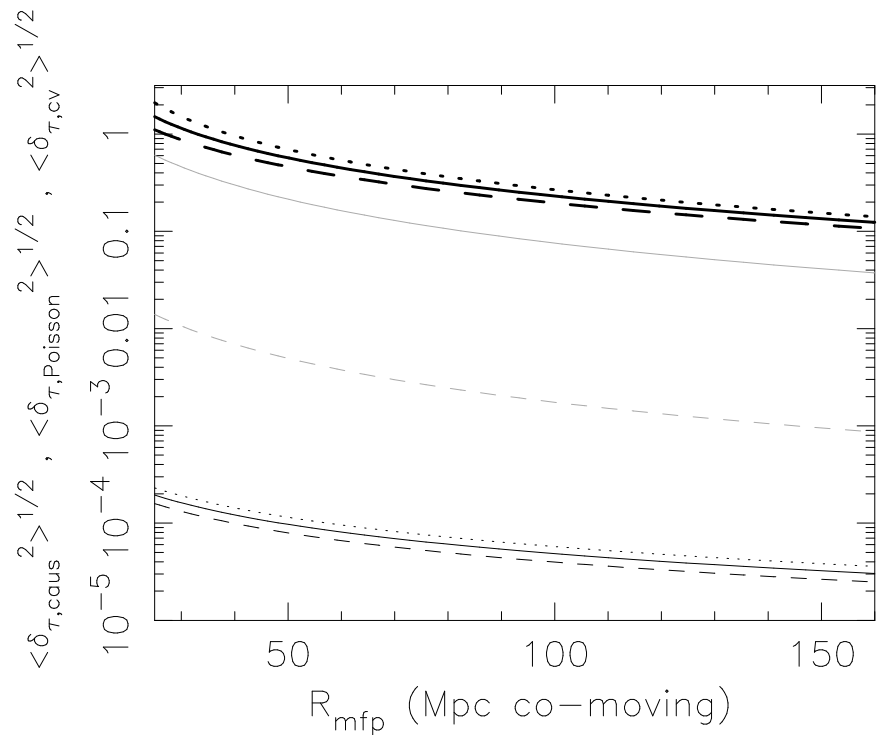

FIG. 2.- Contributions to fluctuations in $\delta_{\tau}$. The thick lines show the fluctuations in optical depth induced through cosmic variance, $\left\langle\delta_{\tau, \mathrm{cv}}^{2}\right\rangle^{1 / 2}$. The dashed, solid and dotted lines correspond to $\gamma=2,3$, and 4 respectively, and for calculation of $F_{\text {col }}$ we have assumed a minimum virial temperature for galaxies of $2 \times 10^{5} \mathrm{~K}$. The values of $\left\langle\delta_{\tau \text {,Poisson }}^{2}\right\rangle^{1 / 2}$ are shown by the light lines; the solid and dashed lines correspond to $t_{1 \mathrm{t}} / t_{\mathrm{H}}=0.01$ and $t_{1 \mathrm{t}} / t_{\mathrm{H}}=0.1$, combined with minimum virial temperatures for galaxies of $2 \times 10^{5} \mathrm{~K}$ and $10^{4} \mathrm{~K}$ respectively. We also show the values of $\left\langle\delta_{\tau, \text { caus }}^{2}\right\rangle^{1 / 2}$ (dark lines with dashed, solid and dotted curves corresponding to $\gamma=2,3$, and 4 respectively). $\left\langle\delta_{\tau, \text { caus }}^{2}\right\rangle^{1 / 2}$ is much smaller than $\left\langle\delta_{\tau, \text { Poisson }}^{2}\right\rangle^{1 / 2}$ and $\left\langle\delta_{\tau, \mathrm{cv}}^{2}\right\rangle^{1 / 2}$. In each case we have evaluated the fluctuations at $z=5.5$.

in $J_{\nu}$. Second we consider $t_{\mathrm{lt}} / t_{\mathrm{H}} \sim 0.1$ corresponding to starburst lifetimes of $\sim 10^{8}$ years (of order the orbital time for a circular orbit in a star-forming disk that occupies $\sim 10 \%$ of the virial radius of its host halo). This lifetime is combined with a minimum halo mass having a virial temperature of $\sim 10^{4} \mathrm{~K}$, corresponding to cooling threshold of atomic hydrogen (so that the filtering scale is assumed not to have had time to respond to the re-heated IGM). This case corresponds to the largest source numbers and hence to the smallest fluctuations in $J_{\nu}$. The Poisson noise in the number of galaxies is $\Delta N_{\mathrm{src}}=\sqrt{N_{\mathrm{src}}}$, so that the fluctuations in the optical depth induced by Poisson fluctuations in the number of sources is

$$
\begin{aligned}
\left\langle\delta_{\tau, \text { Poisson }}^{2}\right\rangle^{\frac{1}{2}} & \sim \sqrt{\left(\frac{\partial \ln \tau}{d N_{\mathrm{src}}} \Delta N_{\mathrm{src}}\right)^{2}}=\sqrt{\left(-\frac{\partial \ln J_{\nu}}{d N_{\mathrm{src}}} \Delta N_{\mathrm{src}}\right)^{2}} \\
& =\sqrt{\left(-\frac{\partial \ln N_{\mathrm{src}}}{d N_{\mathrm{src}}} \Delta N_{\mathrm{src}}\right)^{2}}=\frac{1}{\sqrt{N_{\mathrm{src}}}},
\end{aligned}
$$

where we have used the relation $J_{\nu} \propto N_{\text {src }}$, and evaluated the fluctuation range of $\tau$ corresponding to the 1-sigma level of fluctuations in the number counts of sources. The resulting curves are plotted as a function of $R_{\mathrm{mfp}}$ in Figure 2 .

\subsection{Fluctuations in the optical depth}

The contributions to the redshift variance introduced by causality, by cosmic variance and by source count fluctuations are independent of each other. The positive $(+)$ and negative (-) ranges of allowed $\delta_{\tau}$ may therefore be estimated by adding these contributions in quadrature,

$$
\left\langle\delta_{\tau}^{2}\right\rangle^{\frac{1}{2}}= \pm \sqrt{\left\langle\delta_{\tau, \mathrm{cv}}^{2}\right\rangle+\left\langle\delta_{\tau, \text { caus }}^{2}\right\rangle+\left\langle\delta_{\tau, \text { Poisson }}^{2}\right\rangle} .
$$

\section{RESULTS AND DISCUSSION}

The upper and lower limits that bracket the typical range of $\delta_{\tau}$ at $z=5.5$ are plotted in Figure 3 . The dashed, solid and dotted lines correspond to $\gamma=2,3$ and 4 respectively. The left and right panels show the cases of $t_{\mathrm{lt}} / t_{\mathrm{H}}=0.01$ and 0.1 , combined with minimum galaxy masses corresponding to virial temperatures of $2 \times 10^{5} \mathrm{~K}$ and $10^{4} \mathrm{~K}$ respectively. Figure 3 shows that cosmic variance introduces fluctuations in $\tau$ that are of order unity over a range of length scales. As the mean free path increases the fluctuations become smaller in line with the smaller cosmic variance on large scales. The fluctuations in $J_{\nu}$ introduced by fluctuations in the number of sources provides a small contribution. This is in contrast to the situation for double reionization of helium at lower redshifts where the ionizing sources (quasars) reside in rarer more massive systems (Bolton et al. 2005).

The finding that cosmic variance should result in significant fluctuations in $\tau$ is qualitatively insensitive to variations in the value of $\gamma$ (which parameterizes unknowns regarding the absorber properties) over a wide range. This insensitivity arises partly because the collapsed fraction evolves much more rapidly than a power-law with redshift (because the minimum galaxy mass lies on the exponential tail of the Gaussian density distribution), and partly because the fluctuations are dominated by the $\Delta_{z}^{2}$ term in the evaluation of $\tau$.

We may take the upper limit for $R_{\mathrm{mfp}}$ after reionization to be 120 co-moving Mpc, which is the value measured at $z \sim 4$ (Miralda-Escude et al. 2003), while the lower limit is set by the size of bubbles at the end of the overlap epoch ( $\sim 70$ co-moving Mpc at $z \sim 6$ and larger if reionization completed earlier; Wyithe \& Loeb 2004b). The shaded region in Figure 3 delineates the range for different values of $R_{\mathrm{mfp}}$ assuming $\gamma=3$. We find our main conclusion, 

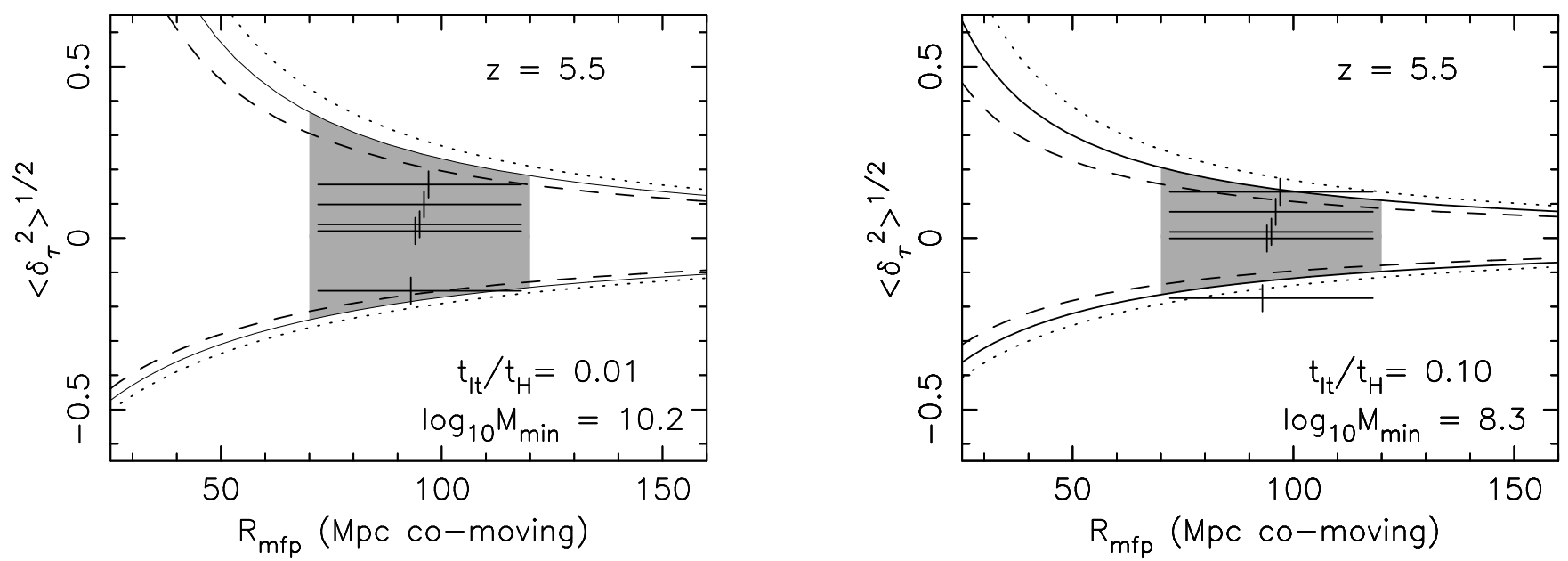

FIG. 3.- The upper and lower values of the 1-sigma range for fluctuations in $\delta_{\tau}$. The dashed, solid and dotted lines correspond to $\gamma=2,3$ and 4 respectively. The grey portion corresponds to the range spanned by different values of $R_{\operatorname{mfp}}$ given $\gamma=3$. The grey region is bounded from the left by the boundary at 70 co-moving Mpc corresponding to the bubble size at the end of then reionization, and from the right by the ionizing photon mean-free-path at $z \sim 4$. We show cases with $z=5.5$, and take populations of ionizing sources having $t_{1 \mathrm{t}} / t_{\mathrm{H}}=0.01$ (left) and 0.1 (right), combined with minimum galaxy masses corresponding to virial temperatures of $\sim 2 \times 10^{5} \mathrm{~K}$ and $\sim 10^{4} \mathrm{~K}$ respectively. The values of $\delta_{\tau}$ at $z \sim 5.6$ measured along the lines-of-sight to five high-redshift quasars (White et al. 2003) are over-plotted. The horizontal error-bars on these observations indicate the possible range of $R_{\mathrm{mfp}}$.

that cosmic variance introduces large fluctuations in the transmission of the IGM at $z \sim 5.5$, holds over this full allowed range of Ly-limit mean-free-paths. The reionization redshift itself is also inhomogeneous, as evidenced by the transmission of flux in the Ly $\alpha$ troughs of some but not all of the $z>6$ quasars (White et al. 2003; Oh \& Furlanetto 2004). Our model becomes applicable at the redshift where most lines of sight probe the post-overlap IGM. Finally, we note that our results do not depend on the reionization redshift having occurred near $z \sim 6$ but apply to earlier reionization epochs as well.

\subsection{Scatter in the transmitted intensity}

The fluctuation in the relative intensity $\left(I \propto e^{-\tau}\right)$ transmitted through the IGM $\left(\delta_{I}=\left(I-I_{\mathrm{av}}\right) / I_{\mathrm{av}}\right)$ is related to the fluctuation in $\tau$ through

$$
\begin{aligned}
\delta_{I} & =\frac{I-I_{\mathrm{av}}}{I_{\mathrm{av}}}=\frac{e^{-\left(\tau_{\mathrm{av}}+\tau_{\mathrm{av}} \delta_{\tau}\right)}-e^{-\tau_{\mathrm{av}}}}{e^{-\tau_{\mathrm{av}}}} \\
& =e^{-\tau_{\mathrm{av}} \delta_{\tau}}-1 .
\end{aligned}
$$

Note that in regions where the optical depth is reduced $\left(\delta_{\tau}<0\right)$ the exponential is positive and the value of $\delta_{I}$ can become very large. For values of optical depth larger than unity the exponential acts as a non-linear amplifier of any inhomogeneities in the IGM which skews the distribution of transmitted intensity. Cosmic variance therefore leads to a highly asymmetric distribution of transmitted flux, even if the distribution of $\delta_{\tau}$ is a symmetric Gaussian. For large values of $\tau \sim 3-5$ immediately following reionization, the variations in the transmission span an order of magnitude. At later times when the optical depth becomes small, equation (18) becomes

$$
\delta_{I} \sim-\tau_{\text {av }} \delta_{\tau} .
$$

Thus at small values of optical depth the distribution of transmitted intensity becomes symmetric.

\subsection{Comparison with observations}

The results presented in Figure 3 may be compared with existing observations of high redshift quasars. Becker et al. (2001) and White et al. (2003) calculated the effective Ly $\alpha$ optical depth in bins of width $\Delta z=0.2$ along the lines of sight to five high redshift quasars. The resulting values of $\delta_{\tau}$ for these five lines of sight in the redshift bin $5.5 \leq z \leq 5.7$ are plotted over the grey region in Figure 3 for comparison. At $z \sim 5.6$ White et al. (2003) find $\tau_{\text {av }} \sim 2.5$ and fractional fluctuations in optical depth with values up to $\left(\tau-\tau_{\mathrm{av}}\right) / \tau_{\mathrm{av}} \sim 0.25$. Since there are only 5 lines of sight we would expect this observed range to be roughly equivalent to the 1 -sigma fluctuations plotted in figure 3 , for which we find $\left\langle\delta_{\tau}^{2}\right\rangle^{\frac{1}{2}} \sim 0.1-0.4$ depending on the value of the mean-free-path and the nature of the ionizing sources. Inspection of Figure 3 therefore suggests that the model is in good agreement with with current data. Djorgovski (2004) has also presented the fluctuations in optical depth along five lines of sight within Gaussian redshift windows of variance $\sigma_{z}=0.05$. This smaller redshift window yields fractional fluctuations in optical depth with values up to $\left(\tau-\tau_{\mathrm{av}}\right) / \tau_{\mathrm{av}} \sim 0.5$. At $z \sim 5.5$, the redshift bin widths of $\Delta z=0.1-0.2$ correspond to co-moving lineof-sight distances $R_{\Delta z}=(1+z) c d t / d z \Delta z \sim 50-100 \mathrm{Mpc}$. Thus the observations sample the IGM over a length-scale comparable to $R_{\mathrm{mfp}}$, and so are fortuitously matched to our calculation. Of course at redshifts where $R_{\mathrm{mfp}}<$ $50-100 \mathrm{Mpc}$, our estimate of $\left\langle\delta_{\tau}^{2}\right\rangle^{\frac{1}{2}}$ may be compared with observation by averaging the predicted fluctuations over a number $\sim\left[(50-100 \mathrm{Mpc}) / R_{\mathrm{mfp}}\right]$ of regions.

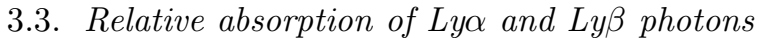

The relative level of absorption for $\operatorname{Ly} \alpha$ and $\operatorname{Ly} \beta$ (or $\mathrm{Ly} \gamma$ etc) photons is dictated by the ratio of the product of oscillator strengths and resonance wavelengths for the two transitions. Assuming a homogeneous IGM this results in a Ly $\alpha$ optical depth that is 6.25 times the $\operatorname{Ly} \beta$ optical depth (Songaila 2004). However the ratio is decreased in an inhomogeneous IGM because on small scales 
transmission is dominated by low-density regions, while the transition with the lower cross-section for absorption is able to sample a larger range of densities. Oh \& Furlanetto (2004) find that values of 2-2.5 are more realistic for the ratio of $\operatorname{Ly} \alpha$ to $\operatorname{Ly} \beta$ optical depth.

The constant of proportionality relating $\tau$ and $J_{\nu}$ will therefore be dependent on the oscillator strength and resonance frequencies. This constant cancels in calculation of $\delta_{\tau}$, and so the range of fractional variation in optical depth will be the same for absorption in $\operatorname{Ly} \alpha$ and $\operatorname{Ly} \beta$. On the other hand the range of optical depth values is given by $\tau_{\mathrm{av}}\left\langle\delta_{\tau}^{2}\right\rangle^{\frac{1}{2}}$. As a result, the absolute range of optical depths observed for Ly $\alpha$ absorption will be larger than the range observed for Ly $\beta$ absorption by a factor of $\sim 2-6$. Similarly, while the relative fluctuations in the optical depth are the same for all transitions, we find that the relative fluctuations in observed intensity $\delta_{I}$ will be much larger for $\operatorname{Ly} \alpha$ than $\operatorname{Ly} \beta$ photons due to the non-linearity of the exponential relation between optical depth and transmission. In particular, if the Ly $\alpha$ optical depth is smaller than unity, then $\delta_{I} \sim-\tau_{\mathrm{av}} \delta_{\tau}$ and the relative fluctuations in intensity may be calculated directly from the ratios of the optical depths among the different transitions. In cases where the Ly $\alpha$ optical depth is larger than unity, the variation in $\delta_{I}$ among the different transitions is larger due to the presence of the exponential in equation (18).

\section{SUMMARY}

In this paper we have calculated the magnitude of the large scale fluctuations in the optical depth to absorption of Ly-series photons that are introduced through variance in the cosmic density field. We find that cosmic variance introduces fractional fluctuations in the optical depth that are of order unity for mean-free paths of ionizing pho- tons $R_{\mathrm{mfp}} \lesssim 150$ co-moving Mpc. This finding explains the variation seen in the Ly $\alpha$ optical depth along different lines of sight through the IGM at $z<6$ (Becker et al. 2001; White et al. 2003).

Our model was not constructed to predict the value of optical depth or to associate a value of the optical depth with the mean-free-path. Rather, it predicts the trend that the relative fluctuations in optical depth will drop as the ionizing photon mean-free-path grows. The mean-freepath increases with cosmic time, and so the range of optical depth will therefore decrease towards low redshift. In addition, while our model predicts a distribution of optical depths that is roughly symmetric about the average, the exponential dependence of transmission on optical depth implies a distribution of transmission that will be highly asymmetric. This asymmetry will become smaller as the average value of optical depth declines at late times. Finally, our model predicts that the absolute fluctuations in the Ly $\alpha$ optical depth should be greater than in the Ly $\beta$ optical depth. This translates to relative fluctuations in the transmission through the IGM that are much larger for $\operatorname{Ly} \alpha$ than for $\operatorname{Ly} \beta$.

As larger numbers of high redshift quasars are discovered through surveys like the Sloan Digital Sky Survey, the statistics of absorption averaged over large scales will be measured along many lines of sight. The results of this paper suggest that the effects of cosmic variance on the post-reionization epoch will be directly observed in these studies.

The authors would like to thank Xiaohui Fan for pointing out an error in an early version of this manuscript. JSBW acknowledges the support of the Australian research council. This work was supported in part by NASA grants NAG 5-13292 and NNG05GH54G for AL.

\section{REFERENCES}

Barkana, R., Loeb, A., 2002, ApJ, 578, 1 2004, ApJ, 601, 64

Baugh, C. M. 1996, MNRAS, 282, 1413

Becker, R. H., et al. 2001, AJ, 122, 2850

Bolton, J. S., Haehnelt, M. G., Viel, M., \& Carswell, R. F. 2005, MNRAS, submitted; astro-ph/0508201

Bond, J.R., Cole, S., Efstathiou, G. \& Kaiser, N., 1991, ApJ, 379, 440

Chen, H.-W., Prochaska, J. X., Bloom, J. S., \& Thompson, I. B. 2005, ApJL, submitted; astro-ph/0508270

Djorgovski, S.G., 2004, in Proc. Tenth Marcel Grossmann Meeting on General Relativity, eds. M. Novello, S. Perez-Bergliaffa and R. Ruffini, astro-ph/0409378

Fan, X., Narayanan, V. K., Strauss, M. A., White, R. L., Becker, R. H., Pentericci, L., \& Rix, H.-W. 2002, AJ, 123, 1247

Fan, X., et al. 2004, AJ, 128, 515

Furlanetto, S. R., Hernquist, L., \& Zaldarriaga, M. 2004, MNRAS, 354,695

Gnedin, N. Y., \& Hui, L. 1998, MNRAS, 296, 44

Iliev, I. T., Shapiro, P. R., \& Raga, A. C. 2005, MNRAS, 361, 405
Iliev, I.T., Mellema, G., Pen, U.-L., Merz, H., Shapiro, P.R., Alvarez, M., 2005, astro-ph/0512187

Kaiser, N., \& Peacock, J. A. 1991, ApJ, 379, 482

Kohler, K., Gnedin, N.Y., Hamilton, A., 2005, astro-ph/0511627

Kohler, K., Gnedin, N. Y., Miralda-Escudé, J., \& Shaver, P. A. 2005, ApJ, 633, 552

Lidz, A., Oh, S.P., Furlanetto, S., 2005, astro-ph/0512427

Mesinger, A., \& Haiman, Z., 2004, ApJL, in press, astro-ph/0406188

Miralda-Escudé, J. 2003, ApJ, 597, 66

Oh, S. P., \& Furlanetto, S. R. 2005, ApJ, 620, L9

Press, W., Schechter, P., 1974, ApJ., 187, 425

Sheth, R. K. \& Tormen, G. 1999, MNRAS, 308, 119

Songaila, A. 2004, AJ, 127, 2598

Spergel, D. N, et al., 2003, Astron. J. Supp., 148, 175

Storrie-Lombardi, L. J., McMahon, R. G., Irwin, M. J., \& Hazard, C. 1994, ApJ, 427, L13

White, R., Becker, R., Fan, X., Strauss, M., 2003, Astron J., 126, 1

Wyithe, J.S.B., Loeb, A., 2004a, Nature, 427, 815

Wyithe, J. S. B., Loeb, A., 2004b, Nature, 432, 194 